\title{
Strategies for Indexed Stock Option Hedgers with Loss-Risk-Minimizing Criterion Based on Monte-Carlo Method
}

\author{
Jianhua Guo ${ }^{1 *}$, Lijuan Deng² \\ ${ }^{1}$ School of Economics and Management, Shaoyang University, Shaoyang, China \\ ${ }^{2}$ Library, Shaoyang University, Shaoyang, China \\ Email: `jhguo888@163.com
}

How to cite this paper: Guo, J. H., \& Deng, L. J. (2019). Strategies for Indexed Stock Option Hedgers with Loss-RiskMinimizing Criterion Based on MonteCarlo Method. Journal of Financial Risk Management, 8, 275-285.

https://doi.org/10.4236/jfrm.2019.84019

Received: November 27, 2019

Accepted: December 14, 2019

Published: December 17, 2019

Copyright () 2019 by author(s) and Scientific Research Publishing Inc. This work is licensed under the Creative Commons Attribution International License (CC BY 4.0).

http://creativecommons.org/licenses/by/4.0/

\section{Open Access}

\begin{abstract}
Unlike traditional options, indexed stock options use market performance as a benchmark reference index, and the option exercise price is a variable that changes with market performance. This paper, by taking the expected loss at the end of the hedging period as a risk measure, conducts a study on the hedging strategies for indexed stock option hedgers. Empirical analysis shows that, firstly, it is more conducive for indexed stock options to play an incentive role by adjusting the exercise price according to changes in market conditions, secondly, when the frequency of hedging position adjustment is relatively high, it can better cope with the price fluctuations in the market, thereby reducing the risk of possible loss and achieving a better hedge effect, but the hedging costs will increase for because of the existence of transaction costs.
\end{abstract}

\section{Keywords}

Indexed Stock Option Hedgers, Loss-Risk-Minimizing, Monte-Carlo Method

\section{Introduction}

All kinds of contingent claims may be perfectly replicated by self-finance strategy if the market is complete, and the cost to replicate is the fair price of the contingent claim; in an incomplete market, investors may also perfectly replicate a contingent claim by super hedging strategy (Bayraktar \& Zhou, 2017), however, with the exception of being costly, super hedging causes the loss of chance to get more profits, thus, many investors are unwilling to do this. In fact, most people only want to pay a small quantity of initial cost to hedge the terminal contingent claim, 
but they have to endure a level of risk, how to find the optimal hedging strategy for such kind of hedging has been a hot topic in finance. Before seeking for the optimal strategy, we should decide a criterion to measure the risk, a simple and exclusively accepted method is the minimal variance hedging (Last \& Penrose, 2011; Makogin, Melnikov, \& Mishura, 2017), even though it's shortcoming to simultaneously punish the profit and the loss; another method to measure risk is VaR (Cong, Tan, \& Weng, 2014; Soloviev, 2016; Capiński, 2015), which anticipates the heaviest lost under given criterion level, however, VaR may be given different value for different investors. As for hedging, only potential shortfall will be considered, with an example European claim, investors' goal is to seek the optimal strategy to minimize the expected loss $E\left(\left[H_{T}-V_{T}(\varphi)\right]^{+}\right)$, which had been originally researched by Follmer \& Leukert (Follmer \& Leukert, 2000), and many subsequently research results have achieved (Kim, 2012; Kabaila \& Mainzer, 2018).

Unlike traditional options, indexed stock options use market performance as a benchmark reference index, and the option exercise price is a variable that changes with market performance, i.e. $K_{T}=S_{0} I_{T} / I_{0}$, where $I_{T}$ represents the market performance, or the overall market trend of the stock market, or the performance of competitors in the same industry at the end of the hedging period, while $S_{0}$ and $I_{0}$ represent the initial price of the underlying stock and the initial market price, respectively. In this way, even in a bull market, a rise in the market will cause the benchmark reference index to rise, and drive the option exercise price to rise, thereby filter the stock price changes in the market due to non-manager efforts, if the performance level of the company is lower than the benchmark index, the value of stock options may still be zero in a bull market, and managers will not receive huge profits when they execute stock options; Conversely, even in a bear market, if the corporate performance is higher than the benchmark index, the option value can also be positive, and the manager can still get incentives for option returns.

This paper, by taking the expected loss i.e. $\min _{\varphi} E\left[H_{T}-V_{T}(\varphi)\right]^{+}$, at the end of the hedging period as a risk measure, and using the Monte Carlo method, conducts a study on the hedging strategies for indexed stock option hedgers. Enlightened by Longstaff and Schwartz (Longstaff \& Schwartz, 2001), Potters M. et al. (Potters, Bouchaud, \& Sestovic, 2001), who priced the option with numerical method, we firstly generate many asset price paths by Monte-Carlos simulation and look on the averaged terminal shortfall as the expected loss, then, basis functions are introduced to estimate hedging positions and finally the optimal strategy is achieved through an algorithm (Seydel, 2017, Monte-Carlo-Simulation $[\mathrm{M}])$.

\section{Some Preliminaries}

Assume there are two kinds of assets: risky asset (Security) and riskless asset (Bond). Let $(\Omega, F, P)$ be a complete probability space with filtration $F=\left(F_{t}\right)_{t \in[0, T]}$, 
and the price of risky asset $S=\left(S_{t}\right)_{t \in[0, T]}$ and the market index (in this paper, which is CSI) $I=\left(I_{t}\right)_{t \in[0, T]}$ be nonnegative and adapted to $F$, satisfying:

$$
\left\{\begin{array}{l}
\mathrm{d} S_{t}=S_{t}\left(\mu \mathrm{d} t+\sigma \mathrm{d} w_{t}+q_{t} \mathrm{~d} N_{t}\right) \\
\mathrm{d} I_{t}=I_{t}\left(\mu^{(I)} \mathrm{d} t+\sigma^{(I)} \mathrm{d} w_{t}^{(I)}+q^{(I)} \mathrm{d} N_{t}^{(I)}\right)
\end{array}\right.
$$

where $w_{t}, w_{t}^{I}$ are standard Brownian motions, $N_{t}, N_{t}^{(I)}$ are Poisson processes with Poisson strength $\lambda, \lambda^{(I)}$ and respectively independent with $w_{t}$ and $w_{t}^{(I)}$, $q^{(I)}, q_{t}>-1$ are amplitudes of price jumps and $J_{t}=\ln \left(1+q_{t}\right) \sim N\left(\mu_{J}, \sigma_{J}^{2}\right)$, $J_{t}^{(I)}=\ln \left(1+q_{t}^{(I)}\right) \sim N\left(\mu_{J}^{(I)},\left(\sigma_{J}^{I}\right)^{2}\right)$.

Let $B=\left(B_{t}\right)_{t \in[0, T]}$ be riskless asset's price process, satisfying:

$$
\mathrm{d} B_{t}=r B_{t} \mathrm{~d} t, r \text { denotes riskless interest rate. }
$$

As for random sequence $l=\left(l_{t}\right)_{t=0,1, \cdots, T}$, let $\Theta(l)$ be a space consisting of all predictable sequences $\left(\vartheta_{t}\right)_{t=0,1, \cdots, T}$ which satisfies $\vartheta_{t} \Delta l_{t} \in L^{2}(P)$.

Call a 2-demension stochastic process $\varphi=\left(\vartheta_{t}, \delta_{t}\right)_{t=0, \cdots, T-1}$ be a investment strategy and $\vartheta_{t} \in \Theta(S), \delta_{t}$ is an adapted process, satisfying:

$$
V_{t}(\varphi):=\vartheta_{t} S_{t}+\delta_{t} B_{t} \in L^{2}(P), \quad t \in\{0,1, \cdots, T-1\},
$$

where $V(\varphi)$ is the value of strategy $\varphi$, when $\left(\vartheta_{t}, \delta_{t}\right)$ denotes the hedging position held at time $t$.

Furthermore, we call it a self-finance strategy when $\varphi=\left(\vartheta_{t}, \delta_{t}\right), t=0, \cdots, T-1$ satisfies:

$$
\vartheta_{t} S_{t+1}+\delta_{t}=\vartheta_{t+1} S_{t+1}+\delta_{t+1}, \quad t=0,1, \cdots, T-1 .
$$

As to strategy $\varphi=\left(\vartheta_{t}, \delta_{t}\right)_{t=0,1, \cdots, T-1}$, we define its shortfall risk as the following:

$$
R_{t}\left(V_{t}, S_{t}, \varphi\right)=E\left\{\left[H_{T}-V_{T}(\varphi)\right]^{+} \mid F_{t}\right\}, t=0,1, \cdots, T-1,
$$

where $V_{t}, S_{t}$ denote the portfolio value and the stock price at time $t$ respectively, $H_{T}=\left(S_{T}-K_{T}\right)^{+}$is a $F_{T}$-measurable and nonnegative random variable which denotes the hedger's payment reliability at the expiration.

Suppose an investor has initially written a share of European Call Option with the exercising price $K$ and $T$ horizon, in order to minimize the terminal shortfall, he hedges the option by self-financing at discrete time $\{0,1, \cdots, T-1\}$ with $V_{0}=\vartheta_{0} S_{0}+\delta_{0}$ as his initial cost, thus, we can express the hedging model as following:

$$
\left\{\begin{array}{l}
\min _{\phi} E\left\{\left[H_{T}-V_{T}(\varphi)\right]^{+}\right\} \\
\text {s.t. } \vartheta_{t} S_{t+1}+\delta_{t} B_{t+1}=\vartheta_{t+1} S_{t+1}+\delta_{t+1} B_{t+1} \\
\quad t=0,1, \cdots, T-1
\end{array} .\right.
$$

\section{Solution}

\subsection{The Parameter-Estimation of the Price Process}

According to the expression (1): 


$$
y_{t}=\left(\mu-\frac{\sigma^{2}}{2}\right)+\sigma \varepsilon_{t}+\sum_{i=N(t-1)+1}^{N(t)} J_{i},
$$

where $\varepsilon_{t}=w_{t}-w_{t-1} \sim N(0,1), Z_{t}=N_{t}-N_{t-1} \sim p(\lambda), \quad J_{i} \sim \operatorname{IIDN}\left(\mu_{J}, \sigma_{J}^{2}\right)$.

Let $\Theta=\left(\mu, \sigma^{2}, \lambda, \mu_{J}, \sigma_{J}^{2}\right), y=\left\{y_{1}, y_{2}, \cdots, y_{T}\right\}, X=\left\{\left(Z_{t}, J_{t}\right)\right\}$ be jump times and jump amplitude respectively, MCMC technology has been used to estimate all parameters, i.e. a Markovian chain of each parameter has been drawn from $p(\Theta, X \mid y) \propto p(y \mid \Theta, X) p(X \mid \Theta) p(\Theta)$ with observations $\left\{y_{1}, y_{2}, \cdots, y_{T}\right\}$ and prior distribution $p(\Theta)$, and averaging the chain as estimator of parameter (Johannes \& Polson, 2006).

With the estimated parameters we can produce:

$$
y_{t}=\ln \left(S_{t} / S_{t-1}\right) \sim N\left(\left(\mu-\frac{\sigma^{2}}{2}\right)+\lambda \mu_{J}, \sigma^{2}+\lambda\left(\sigma_{J}^{2}+\mu_{J}^{2}\right)\right) .
$$

\subsection{The Monte-Carlo Simulation of Price Process}

Let $f\left(x_{1}, \cdots, x_{n}\right)$ be the union density function of random vector $\left(X_{1}, \cdots, X_{n}\right)$, $f\left(x_{i+1} \mid x_{1}, \cdots, x_{i}\right)$ be conditional density function of $X_{i+1}$ with $X_{1}, \cdots, X_{i}$ being known, according to the Bayesian theory:

$$
f\left(x_{1}, \cdots, x_{n}\right)=f\left(x_{1}\right) f\left(x_{2} \mid x_{1}\right) \cdots f\left(x_{n} \mid x_{1}, \cdots, x_{n-1}\right) .
$$

Theoretically, we can draw $\left(X_{1}, \cdots, X_{n}\right)$ from the union density function $f\left(x_{1}, \cdots, x_{n}\right)$, but in fact, we do the following:

Firstly, drawing $\tilde{X}_{1} \sim f\left(x_{1}\right)$; then, $\tilde{X}_{2} \sim f\left(x_{2} \mid \tilde{X}_{1}\right)$ and so on, drawing $\tilde{X}_{n} \sim f\left(x_{n} \mid \tilde{X}_{1}, \cdots, \tilde{X}_{n-1}\right)$, and we can deduce that the drawn sequence $\left(\tilde{X}_{1}, \cdots, \tilde{X}_{n}\right)$ has a union density function $f\left(x_{1}, \cdots, x_{n}\right)$.

\subsection{Strategy Decision}

On discrete time $\{0,1, \cdots, T-1\}$, assuming Bond's price $B \equiv 1$, the stock price has been discounted. Under the constraint of self-financing (4), we have:

$$
\begin{aligned}
V_{T} & =V_{T-1}+\vartheta_{T-1} \Delta S_{T-1} \\
& =V_{T-2}+\vartheta_{T-2} \Delta S_{T-2}+\vartheta_{T-1} \Delta S_{T-1}, \\
& =\cdots=V_{0}+\sum_{t=0}^{T-1}\left(\vartheta_{t} \Delta S_{t}\right)
\end{aligned}
$$

where $\Delta S_{t}=S_{t+1}-S_{t}, t=0,1, \cdots, T-1, V_{0}$ is the initial cost. Now, substituting (6) with (9), the optimizing problem (6) becoming into:

$$
\min _{\varphi} E\left\{\left[H_{T}-V_{0}-\sum_{t=0}^{T-1}\left(\vartheta_{t} \Delta S_{t}\right)\right]^{+}\right\},
$$

Up to now, our goal is to find a self-financing strategy for optimization problem (10), however, it is a stochastic programming problem, and $H_{T}-V_{0}-\sum_{t=0}^{T-1}\left(\vartheta_{t} \Delta S_{t}\right)$ depends on the whole price path, so, it is difficult to directly solve (10). Having generated $M$ independent price paths by Monte-Carlo simulation method, solving problem (10) is equivalent to solving the following optimizing problem: 


$$
\min _{\varphi} \sum_{m=1}^{M}\left[H_{T}^{(m)}-V_{0}-\sum_{t=0}^{T-1}\left(\vartheta_{t}^{(m)} \Delta S_{t}^{(m)}\right)\right]^{+} .
$$

Obviously, the unknown variables in (11) equal $M \cdot T$, where $M$ denotes the number of scenarios and $T$ denotes the adjusting frequency, thus, it is computationally challenging to directly solve problem (11) when the number of scenarios is large and the adjusting is frequent. In order to simplify (11), we try to approximate holdings $\vartheta_{t}$ by basis functions, having done this, the number of unknowns at each hedging time is reduced to the number of parameters in the basis functions, which is typically very small.

Assume that the holding $\vartheta_{t}$ is decided by the underlying stock price at any time $t$, i.e. $\vartheta_{t}=\vartheta\left(S_{t}\right)$, by definition $1, \vartheta_{t} \Delta S_{t} \in L^{2}(P)$, and the Hilbert space $L^{2}(P)$ is of countable orthonormal basis, so, the holding $\vartheta_{t}$ can be linearly represented by basis functions (Potters, Bouchaud, \& Sestovic, 2001):

$$
\vartheta_{t}=\vartheta\left(S_{t}\right)=\sum_{j=1}^{p} a_{j}^{(t)} L_{j}\left(S_{t}\right), t=0,1, \cdots, T-1,
$$

where $L_{j}\left(S_{t}\right)$ denotes basis function and $a_{j}^{(t)}$ denotes the corresponding constant coefficients, $p$ is the number of selected basis functions such as Hermite polynomial, Legendre polynomial, Chebyshev polynomial, Laguerre polynomial, hereafter we choose Laguerre polynomial as basis function which is formed as following:

$$
L_{n}(x)=\exp \left(-\frac{x}{2}\right) \frac{\exp (x)}{n !} \frac{\mathrm{d}^{n}}{\mathrm{~d} x^{n}}\left(x^{n} \mathrm{e}^{-x}\right)
$$

After substituting (12) into (11), the optimizing problem (17) changes into:

$$
\min _{\varphi} \sum_{m=1}^{M}\left[H^{(m)}-V_{0}-\sum_{t=0}^{T-1}\left(\left(\sum_{j=1}^{p} a_{j}^{(t)} L_{j}\left(S_{t}^{(m)}\right)\right) \Delta S_{t}^{(m)}\right)\right]^{+} .
$$

Comparing (13) with (11), we find the number of unknowns has greatly decreased, and the holding $\vartheta_{t}$ may be deduced by (12) only with the selected basis functions and the simulated price scenarios, which is solved by the steepest descent method.

\subsection{The Steepest Descent (Wahab \& Khan, 2018)}

Assuming objective function $f(x), x \in R^{n}$, when $\nabla f\left(x_{k}\right) \neq 0$, at $x_{k}$, expressing $f(x)$ with:

$$
f(x)=f\left(x_{k}\right)+\nabla f\left(x_{k}\right)^{\prime}\left(x-x_{k}\right)+o\left(\left\|x-x_{k}\right\|\right) .
$$

Denoting $x-x_{k}=\alpha d_{k}(\alpha>0)$, then the expression (20) can be expressed:

$$
f\left(x_{k}+\alpha d_{k}\right)=f\left(x_{k}\right)+\alpha \nabla f\left(x_{k}\right)^{\prime} d_{k}+o\left(\left\|\alpha d_{k}\right\|\right) .
$$

We call $d_{k}$ the descent direction of $f(x)$ when $\nabla f\left(x_{k}\right)^{\prime} d_{k}<0$. As to small $\alpha$, there is $f\left(x_{k}+\alpha d_{k}\right)<f\left(x_{k}\right)$, and with smaller $\nabla f\left(x_{k}\right)^{\prime} d_{k}, f(x)$ has greater descent at $x_{k}$. denoting $\theta_{k}$ as the plane included angle of 
$-\nabla f\left(x_{k}\right)^{\prime}$ and $d_{k}$, by $-\nabla f\left(x_{k}\right)^{\prime} d_{k}=\left\|\nabla f\left(x_{k}\right)\right\| \cdot\left\|d_{k}\right\| \cos \theta_{k}$, when $\theta_{k}=0$, i.e., $d_{k}=-\nabla f\left(x_{k}\right), \nabla f\left(x_{k}\right)^{\prime} d_{k}$ arrives at the smallest value, and $-\nabla f\left(x_{k}\right)$ is called as the steepest descent direction, $d_{k}=-\nabla f\left(x_{k}\right)$ is the optimal searching direction. The iterative format of the steepest descent method is as following:

$$
x_{k+1}=x_{k}+\alpha_{k} \nabla f\left(x_{k}\right),
$$

where $\alpha_{k}$ denotes the step length decided by linear searching method.

The process of the steepest descent method is as following:

Step 1: Given the initial $x_{0} \in R^{n}$ and the terminating error $\varepsilon>0$, set $k:=0$;

Step 2: Calculating $d_{k}=-\nabla f\left(x_{k}\right)$, stop when $\left\|d_{k}\right\|<\varepsilon$ and $x_{k}$ is the optimal solution;

Step 3: Deciding the step length $\alpha_{k}$ by the linear searching method;

Step 4: Setting $x_{k+1}=x_{k}+\alpha_{k} \nabla f\left(x_{k}\right), k:=k+1$, transfer to the step 2 .

\section{Numerical Example}

\subsection{The Parameter Deciding of the Price Model}

In this subject, we sampled 11,598 high frequency history data of Shanghai Securities Complex Index and the stock ICBC from the 2nd, January to the 28th, December, 2018, and estimated the jump-diffusion process's parameters in WinBugs1.4 by Monte Carlo technology, the estimating results are expressed in Table 1.

\subsection{The Simulated Scenarios and Strategies}

Firstly, according to the parameter values in Table 1, we simulated $M=10000$ price scenarios for the underlying asset by the Monte-Carlo technology submitted in subsection 2.1; Then, deciding the number of basis function, since the computation result does not obviously improve when the number of basis function is more than 3, we choose the first 3 Lagurre polynomials as basis function to approximate the holding position; Finally, in the light of the optimization model (12), the optimal holdings are acquired through numerical algorithm presented in subsection 2.4 with the Matlab software.

\subsection{Analyzing Results}

We assume that a hedger has written the 1-month and 3-month expiration indexed stock option based on the stock ICBC at the 28th, December, 2018, in order to minimize the terminal expected loss, he hedges the contingent claim with stock ICBC and Bond by self-financing with daily, weekly and biweekly

Table 1. parameter valuation of the jump-diffusion process (Data source, http://quotes.money.163.com/stock).

\begin{tabular}{cccccc}
\hline parameters & $\mu\left(\mu^{(I)}\right)$ & $\sigma\left(\sigma^{(I)}\right)$ & $\mu_{J}\left(\mu_{J}^{(I)}\right)$ & $\sigma_{J}\left(\sigma_{J}^{(I)}\right)$ & $\lambda\left(\lambda^{(I)}\right)$ \\
\hline Underlying tock (S) & 0.0025 & 0.0411 & 0.0183 & 0.0522 & 0.0490 \\
Index (I) & 0.0023 & 0.019 & 0.0155 & 0.0519 & 0.0453 \\
\hline
\end{tabular}


rebalancing frequency, the initial Index price and ICBC price are $I_{0}=2493.9$, $S_{0}=5.29$, respectively, and the riskless interest rate is $0.30 \%$ equal to the current savings interest rate offered by the $\mathrm{PBC}$ in December, 2018

(http://www.pbc.gov.cn/), the transaction fee rate $f=0.001,0.002,0.004$, respectively.

As for all hedging cases, we calculate:

Total risk:

$$
\left(H_{T}-V_{T}\right)^{+}
$$

Total cost:

$$
V_{0}+\vartheta_{0} f S_{0}+\sum_{t=1}^{T-1}\left|\vartheta_{t}-\vartheta_{t-1}\right| f S_{t}+\vartheta_{T-1} f S_{T}+H_{T}-V_{T},
$$

where $V_{0}$ is the initial cost; $V_{T}$ denotes the terminal portfolio valuation.

Substituting (12) into (18):

Total cost:

$$
\vartheta_{0} f S_{0}+\sum_{t=1}^{T-1}\left|\vartheta_{t}-\vartheta_{t-1}\right| f S_{t}+\vartheta_{T-1} f S_{T}+H_{T}-\sum_{t=0}^{T-1}\left(\vartheta_{t} \Delta S_{t}\right) .
$$

As a matter of fact, with the exception $V_{0}$, transaction fee and the terminal $H_{T}$, no other hedging cost is required.

The averaged hedging cost and expected loss with different striking price and different strategy adjustment frequency are calculated in Table 2 and Table 3 with 10,000 price simulations.

Firstly, because the price fluctuation will be heavier if option's expiration is longer, a European indexed stock option hedger may be faced with higher risk and must invest more to hedge possible loss risk, for example, in Table 3, the averaged hedging costs (with 3-month expiration) for all kinds of strategy adjustment frequencies and all kinds of transaction fee rates are more than those in Table 2 (with 1-month expiration), i.e., for daily hedging strategy adjustment frequency, 1.5011 is bigger than 1.3251, 2.7656 is bigger than 2.4930, 5.2962 is bigger than 4.6254, and for weekly and biweekly hedging strategy adjustment frequencies, there are similar results. Other more, We can also see that the expected loss may be smaller when the hedging strategy adjusting time step is shorter, for example, in Table 2, 0.702 is the least expected loss, which corresponds to daily hedging strategy adjustment frequency, while 0.8010 is the biggest expected loss, which corresponds to biweekly hedging strategy adjustment frequency, similar results in Table 3.

In addition, we can know by the expression $\left(H_{T}-V_{T}\right)^{+}=\left(\left(S_{T}-K_{T}\right)^{+}-V_{T}\right)^{+}$ that the higher the striking price is, the smaller the expected loss may be; and it is more impossible for the indexed stock option with higher striking price to be executed, which results in decreased hedging cost. In fact, Table 2 and Table 3 indicate the reverse relationship between expected loss with striking price, and the following Figure 1, indicating the relationship between the holding position at the middle time point with the terminal exercising price for the indexed stock 
Table 2. Averaged hedging cost and expected loss with 1-month expiration over 10,000 scenarios $\left(K_{T}=S_{0} I_{T} / I_{0}=5.29 \times 2584.57 / 2493.9=5.48\right)$.

\begin{tabular}{|c|c|c|c|c|c|c|c|}
\hline \multirow[b]{2}{*}{ f } & \multirow{2}{*}{ frequency } & \multicolumn{2}{|c|}{ Daily } & \multicolumn{2}{|c|}{ Weekly } & \multicolumn{2}{|c|}{ Biweekly } \\
\hline & & Expected loss & cost & Expected loss & cost & Expected loss & cost \\
\hline & 0.001 & 0.0702 & 1.3251 & 0.0731 & 1.0031 & 0.0810 & 1.0005 \\
\hline & 0.002 & 0.0702 & 2.4930 & 0.0731 & 1.9821 & 0.0810 & 1.8964 \\
\hline & 0.004 & 0.0702 & 4.6254 & 0.0731 & 3.6987 & 0.0810 & 3.5102 \\
\hline
\end{tabular}

Table 3. Averaged hedging cost expected loss with 3-month expiration over 10,000 scena$\operatorname{rios}\left(K_{T}=S_{0} I_{T} / I_{0}=5.29 \times 3090.76 / 2493.9=6.56\right)$.

\begin{tabular}{|c|c|c|c|c|c|c|c|}
\hline \multirow[b]{2}{*}{$\mathrm{f}$} & \multirow[t]{2}{*}{ frequency } & \multicolumn{2}{|c|}{ Daily } & \multicolumn{2}{|c|}{ Weekly } & \multicolumn{2}{|c|}{ Biweekly } \\
\hline & & Expected loss & cost & Expected loss & cost & Expected loss & cost \\
\hline & 0.001 & 0.0508 & 1.5011 & 0.0526 & 1.2513 & 0.0529 & 1.2120 \\
\hline & 0.002 & 0.0508 & 2.7656 & 0.0526 & 2.3111 & 0.0529 & 2.2589 \\
\hline & 0.004 & 0.0508 & 5.2962 & 0.0526 & 4.4596 & 0.0529 & 4.3254 \\
\hline
\end{tabular}

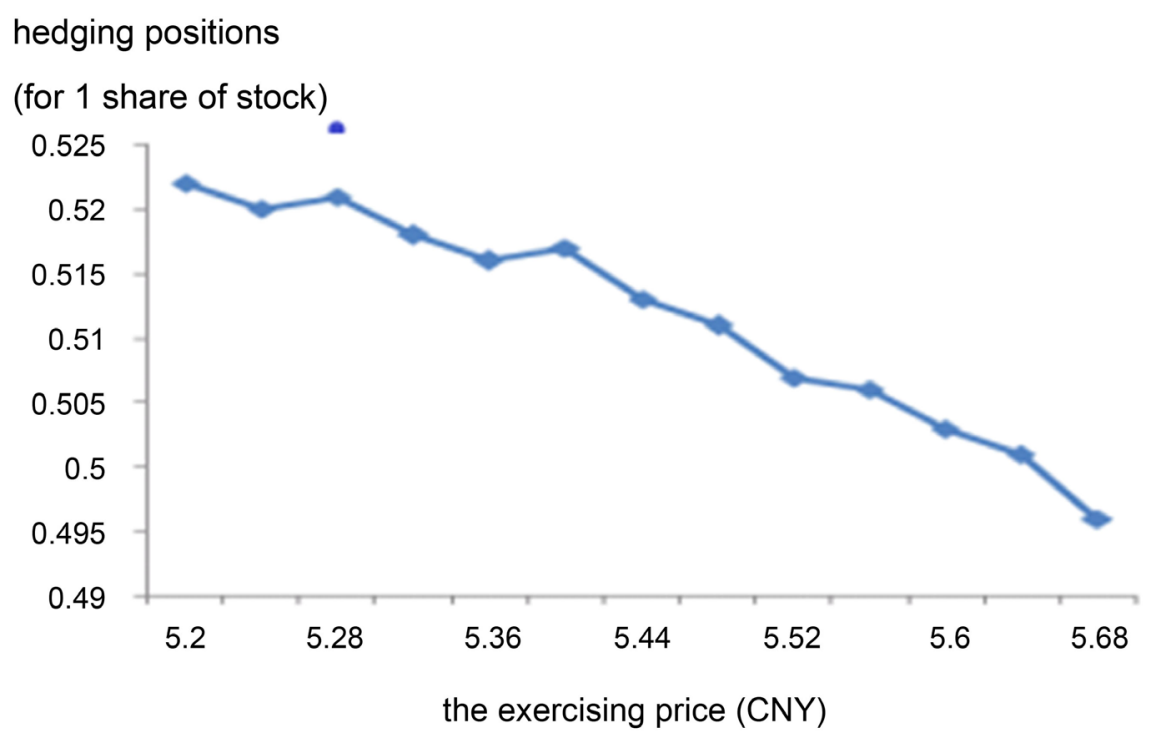

Figure 1. Relationship between holdings and striking prices at middle time point.

option with single month expiration. In Figure 1, the horizontal axis data shows the end-of-period execution prices, while the data on the vertical axis represent the hedging positions that need to be held for 1 share of stock to be hedged at the middle time point with 1-month hedging period. Taking the hedging practice for the ICBC stock as an example, if the terminal exercising price is 5.2CNY, the optimal hedging position is 0.522 shares of stock index futures contract, while the terminal exercising price is $5.68 \mathrm{CNY}$, the optimal hedging position is 0.496 shares of stock index futures contract.

Figure 2 illustrates the relationship between holding positions and different executing prices for 1-month time limit indexed stock option with daily hedging 


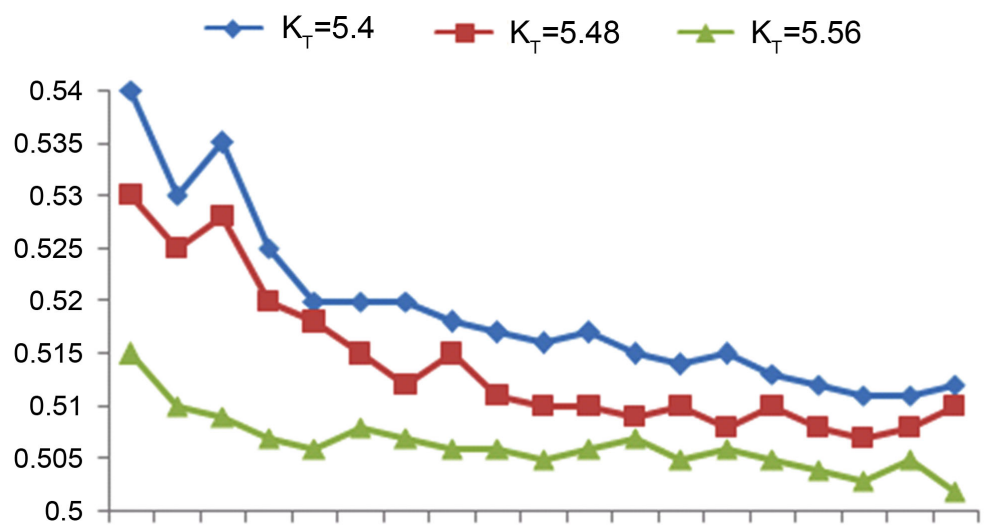

Figure 2. Monthly adjusting position plot with different striking prices.

strategy adjusting frequency. As a whole, the reverse relationship between holding position with striking price can still be found; what's more, the three dash dot lines denoting holding position changing in Figure 2 all rightward incline, which explains that the required holding position may be decreased with time's going by. it is well known that the indexed stock option will be executed at the maturity date, because the farer away the maturity date is, the more heavily the underlying asset's price fluctuates, therefore, more hedging cost must be invested to acquire the same hedging efficiency.

Finally, it is obvious that the lowest line in Figure 2 fluctuate mildly, especially when $K_{T}$ equals to 5.56, the curve almost fluctuates around a line, however, the upper two curves denoting $K=5.4,5.48$, the option being in the money, fluctuate more heavily than the lowest curve, the option being out of the money, which correspond to the third column in Table 2, the hedging cost of option in the money augments more heavily than option out of the money corresponding to the augment of striking price $K_{T}$.

\section{Conclusion}

It is well-known that the goal of hedging is to decrease the risk arising from the price fluctuating, the core objective of hedging is to ascertain reasonable hedging strategies. In this paper, we construct the optimizing model to minimize the terminal shortfall risk under the constraint of self-financing, by Monte-Carlo simulation, many price scenarios are generated and are averaged to estimate the expected shortfall, then, basis functions are imported to approximate the holding positions, finally, the optimal hedging positions are acquired by numerical technology. Table 2, Table 3, Figure 1 and Figure 2 indicate: the technique put forward in this paper is feasible and valuable for investors to hedge risk.

1) Table 2 and Table 3 illustrate, the higher the hedging strategy adjusting frequency is, the more superior the hedging efficiency is.

2) Figure 1 and Figure 2 indicate, the holding position is in inverse proportion to the striking price, i.e., the higher executing price the European call option has, the lower holding position may be held, vice versa. In this way, we can hedge 
risk and save cost at the same time.

In conclusion, because the market is changing rapidly, in order to obtain better hedging results, it is necessary to make reasonable adjustments for hedging positions based on market changes. In other words, frequent hedging strategy adjustments can reduce period-end losses, but because of the existence of transaction costs, excessively frequent hedging strategy adjustments may not be desirable, conversely, if the adjustment frequency of the hedging strategy is too low, it is difficult to achieve expected hedging effect.

Relative to existing research results, in this paper, there are two innovations and main contribution, the first is to expand the application of indexed stock options in the field of hedging, the second is to propose a solution for the nonlinear optimization problem (As shown in expression 6). However, the relevant conclusions of this study are all based on simulation data. Whether different simulated data have influence on the conclusion has not been explained theoretically. This is also our future research direction.

\section{Acknowledgements}

The study was supported by the Hunan provincial social science fund project of China (Project Number: 17YBA354).

\section{Conflicts of Interest}

The authors declare no conflicts of interest regarding the publication of this paper.

\section{References}

Bayraktar, E., \& Zhou, Z. (2017). Super-Hedging American Options with Semi-Static Trading Strategies under Model Uncertainty. International Journal of Theoretical and Applied Finance (IJTAF), 20, 1-8. https://doi.org/10.1142/S0219024917500364

Capiński, M. J. (2015). Hedging Conditional Value at Risk with Options. European Journal of Operational Research, 242, 688-691. https://doi.org/10.1016/j.ejor.2014.11.011

Cong, J. F., Tan, K. S., \& Weng, C. G. (2014). Conditional Value-at-Risk-Based Optimal Partial Hedging. Journal of Risk, 16, 49-83. https://doi.org/10.21314/JOR.2014.293

Follmer, H., \& Leukert, P. (2000). Efficient Hedging: Cost versus Shortfall Risk. Finance and Stochastics, 4, 117-146. https://doi.org/10.1007/s007800050008

Johannes, M., \& Polson, N. (2006). MCMC Methods for Continuous-Time Financial Econometrics. In Y. Aït-Sahalia, \& L. P. Hansen (Eds.), Handbook of Financial Econometrics, Amsterdam: Elsevier Science.

Kabaila, P., \& Mainzer, R. (2018). Estimation Risk for Value-at-Risk and Expected Shortfall. Journal of Risk, 20, 29-47. https://doi.org/10.21314/JOR.2018.376

Kim, J. H. (2012). Shortfall Risk Minimization: The Dual Approach. The Pure and Applied Mathematics, 19, 179-192. https://doi.org/10.7468/jksmeb.2012.19.2.179

Last, G., \& Penrose, M. D. (2011). Martingale Representation for Poisson Processes with Applications to Minimal Variance Hedging. Stochastic Processes \& Their Applications, 121, 1588-1606. https://doi.org/10.1016/j.spa.2011.03.014 
Longstaff, F. A., \& Schwartz, E. S. (2001). Valuing American Options by Simulation: A Simple Least-Squares Approach. The Review of Financial Studies, 14, 113-147. https://doi.org/10.1093/rfs/14.1.113

Makogin, V., Melnikov, A., \& Mishura, Y. (2017). On Mean-Variance Hedging under Partial Observations and Terminal Wealth Constraints. International Journal of Theoretical \& Applied Finance, 20, 1-21. https://doi.org/10.1142/S0219024917500315

Potters, M., Bouchaud, J. P., \& Sestovic, D. (2001). Hedged Monte-Carlo: Low Variance Derivative Pricing with Objective Probabilities. Physica A: Statistical Mechanics and Its Applications, 289, 517-525. https://doi.org/10.1016/S0378-4371(00)00554-9

Seydel, R. U. (2017). Monte-Carlo-Simulation. Berlin: Springer Berlin Heidelberg. https://doi.org/10.1007/978-3-662-50299-0_3

Soloviev, A. I. (2016). Minimax Estimation of Value-at-Risk under Hedging of an American Contingent Claim in a Discrete Financial Market. Contributions to Game Theory and Management, 9, 276-286.

Wahab, A., \& Khan, S. (2018). Comments on "Fractional Extreme Value Adaptive Training Method: Fractional Steepest Descent Approach". IEEE Transactions on Neural Networks and Learning Systems, 1-3. https://doi.org/10.1109/TNNLS.2019.2899219 